\title{
Periodic solution and control optimization of a prey-predator model with two types of harvesting
}

\author{
Jianmei Wang ${ }^{1}$, Huidong Cheng ${ }^{1 *}$, Hongxia Liu' ${ }^{1,2}$ and Yanhui Wang ${ }^{1,2}$
}

"Correspondence:

chd900517@sdust.edu.cn

${ }^{1}$ College of Mathematics and

Systems Science, Shandong

University of Science and

Technology, Qingdao, China

Full list of author information is

available at the end of the article

\section{Springer}

\begin{abstract}
In this work, a prey-predator model with both state-dependent impulsive harvesting and constant rate harvesting is investigated, where the replenishment rate of prey and the harvesting rate are linearly related with the selected threshold. By first using the successor function method and differential equation geometry theory, the existence, uniqueness and asymptotic stability of the order-1 periodic solution are discussed. And then numerical simulations with an example are given to illustrate the feasibility of the theorem-related results. Moreover, in order to increase the total profit, the optimization strategy is presented and the optimal threshold is obtained.
\end{abstract}

MSC: 34C25; 34D20; 92B05; 34A37

Keywords: prey-predator model; state-dependent impulse; order-1 periodic solution; optimization; stability

\section{Introduction}

Fishery is the natural source and basis of fishery production, and it is also one of the important food sources for human beings. If fishery resources are used properly, it can adapt to the natural regeneration ability of the resource and maintain the optimum sustainable yield. If people harvest fish unrestricted, it will lead to the extinction of the species [1-4]. Therefore, looking for a reasonable harvest strategy to ensure the sustainable development of fishery resources has become the focus of research.

In the past few decades, various harvest strategies have been proposed and implemented in fishing industry. In general, if a species is harvested frequently and regularly, we can adopt the strategy with constant rate harvesting [5-8]. And due to the seasonal and economic reasons, periodic harvesting is an effective harvesting strategy for the infrequent harvesting. This periodic harvesting can be described by impulsive differential equations [9-16]. There are some papers studying the effects of periodic impulse harvesting strategy to the species resource. For example, Pei et al. [17] proposed a continuous impulsive harvesting strategy for a prey-predator system with stage structure and time delay, and analysed the global attractivity of extinction periodic solution of the mature predator. Jiao et al. [18] considered a periodic impulsive harvesting prey-predator system with prey hibernation, and they obtained the conditions of the global asymptotic stability criterion for the predator-extinction boundary and the permanent conditions. However, these two

(c) The Author(s) 2018. This article is distributed under the terms of the Creative Commons Attribution 4.0 International License (http://creativecommons.org/licenses/by/4.0/), which permits unrestricted use, distribution, and reproduction in any medium, provided you give appropriate credit to the original author(s) and the source, provide a link to the Creative Commons license, and indicate if changes were made. 
methods of harvesting are carried out without knowing the number of species, which can lead to overexploitation and even depletion of resources.

Recently, state-dependent impulse feedback control has attracted the attention of many scholars [19-23], a novel strategy based on state-dependent impulse feedback control is proposed and applied in the harvest [24-27] and pest management [28-33]. The procedure goes like this: when the number of species reaches a specific requirement, the harvesting strategy is implemented, otherwise the harvesting behavior is suppressed. Some other related studies can be found in [34-39] and the references therein.

Brauer and Soudack [5] considered the following prey-predator system with constant rate harvest for predator:

$$
\left\{\begin{array}{l}
x^{\prime}=x f_{1}(x, y), \\
y^{\prime}=y f_{2}(x, y)-H,
\end{array}\right.
$$

and analyzed the asymptotically stable interval of the system under different cases, where $f_{1}(x, y), f_{2}(x, y)$, respectively, are the average growth rate of $x$ and $y$. After further research on renewable resources, Huang et al. [40,41] combined constant rate harvesting with state-dependent impulse harvesting, and introduced a real-time monitoring system, further improved the harvesting approach. And they proved the existence, uniqueness and stability of periodic solution. However, when implementing impulse strategy, they only considered the harvest of the predator, while ignoring that the prey was also harvested at the same time, and the control parameters were not linearly related to the threshold. Therefore, for the system (1), we introduce the state-dependent impulse fishing strategy, assuming that predators are produced by supplementing preys, let $f_{1}(x, y)=a\left(1-\frac{x}{K}\right)-b y$, $f_{2}(x, y)=\lambda b x-d$, we obtain the following impulsive differential system:

$$
\left\{\begin{array}{l}
x^{\prime}(t)=a x(t)\left(1-\frac{x(t)}{K}\right)-b x(t) y(t), \\
y^{\prime}(t)=y(t)(\lambda b x(t)-d)-H, \\
\Delta x(t)=-p(x) x(t)+\tau(x), \\
\Delta y(t)=-q(x) y(t),
\end{array}\right\} \quad x>h,
$$

where

$$
\left\{\begin{array}{l}
p(x)=p_{\max }-\left(p_{\max }-p_{\min }\right) \frac{x-h_{\min }}{h_{\max }-h_{\min }}, \\
\tau(x)=\tau_{\max }-\left(\tau_{\max }-\tau_{\min }\right) \frac{x-h_{\min }}{h_{\max }-h_{\min }}, \\
q(x)=q_{\max }-\left(q_{\max }-q_{\min }\right) \frac{x-h_{\min }}{h_{\max }-h_{\min }},
\end{array}\right.
$$

$x(t)$ and $y(t)$ refer to the prey fish and predator fish densities at time $t . a>0$ and $K>0$ denote the intrinsic birth rate and the carrying capacity for the prey fish when $y \equiv 0$, respectively. $b>0$ is the predation coefficient, $0<\lambda<1$ represents the conversion coefficient, and $H>0$ denotes the constant harvesting rate of predator fish. $h>0$ is a threshold. When the prey fish density is greater than $h$, i.e., $x>h$, it shows that the prey fish is sufficient and there is no need for impulse control. If the prey fish density decreases to $h$, i.e., $x=h$, the ecological balance of the fishery will be in disorder, we must replenish the prey fish at the replenishment rate $\tau>0$, and harvest the predator fish at rate $q \in(0,1)$, we also harvest 
the prey fish at rate $p \in(0,1)$ while harvesting the predator fish. The control parameters $p(x), \tau(x)$ and $q(x)$ are continuous functions defined on $\left[h_{\min }, h_{\max }\right]$ (see [42]), where $h_{\min }$ and $h_{\max }$ are, respectively, the minimum value and maximum value of the threshold which satisfy $0<h_{\min } \leq h \leq h_{\max }<\frac{K-\tau_{\min }}{1-p_{\min }}$. Furthermore, $p\left(h_{\max }\right)=p_{\min }, p\left(h_{\min }\right)=p_{\max }$, $\tau\left(h_{\max }\right)=\tau_{\min }, \tau\left(h_{\min }\right)=\tau_{\max }, q\left(h_{\max }\right)=q_{\min }$ and $q\left(h_{\min }\right)=q_{\max }$. Denote $p_{h}=p(h), \tau_{h}=\tau(h)$ and $q_{h}=q(h)$.

The main contents of this work are organized as follows. In Section 2, some main definitions and lemmas are provided. In Section 3, the existence, uniqueness and asymptotic stability of the order-1 periodic solution of system (2) are mainly discussed under some conditions. The theoretical results are then verified by numerical simulations, and the optimization problem is presented and solved for obtaining the maximum harvesting profits in Section 4. This work ends with a conclusion.

\section{Preliminaries}

Definition 2.1 ([43]) Consider the general differential system with state-dependent impulse

$$
\left\{\begin{array}{l}
x^{\prime}(t)=P(x, y), \\
y^{\prime}(t)=Q(x, y), \\
\triangle x(t)=\alpha(x, y), \\
\triangle y(t)=\beta(x, y),
\end{array}\right\} \quad(x, y) \notin M,
$$

The dynamic system constituted by the solution mappings of system (4) is called a semicontinuous dynamic system, which is denoted as $(\Omega, g, I, M)$. Let the initial point $A \in \Omega=$ $R_{+}^{2} \backslash M$, and the function $I$ is a continuous impulse mapping that satisfies $I: M \rightarrow N$. $M$ and $N$ are, respectively, the impulsive set and the phase set which represent the curves or straight lines in the plane $R_{+}^{2}$.

Remark 2.1 Based on system (2), we get $M=\{(x, y) \mid x=h, y \geq 0\}, N=\{(x, y) \mid x=(1-$ $\left.\left.p_{h}\right) h+\tau_{h}, y \geq 0\right\}$, for any point $(x, y) \in M$, when $x=h$, we get $I:(h, y) \in M \rightarrow\left(\left(1-p_{h}\right) h+\right.$ $\left.\tau_{h},\left(1-q_{h}\right) y\right) \in N$. For this article, the coordinate of the arbitrary point $A \in R_{2}^{+}$is marked as $\left(x_{A}, y_{A}\right)$.

Definition 2.2 ([44]) If there exist a point $A \in N$ and a time $T$ such that $g(A, T)=B \in M$ and $I(B)=I(g(A, T))=A \in N$, then $g(A, t)$ is defined as an order-1 periodic solution of system (4) with period $T$.

Definition $2.3([45])$ Assume $\Gamma=g(A, t)$ is an order-1 periodic solution of system (4). The order-1 periodic solution $\Gamma$ is orbitally asymptotically stable if for any $\varepsilon>0$, there must exist $\delta>0$ and $t_{0} \geq 0$, such that, for any point $A_{1} \in U(A, \delta) \cap N$ and $t>t_{0}$, we have $\rho\left(g\left(A_{1}, t\right), \Gamma\right)<\varepsilon$.

Definition 2.4 ([46]) Assuming that the impulse set $M$ and the phase set $N$ are both straight lines; see Figure 1. For any point $B_{1} \in N$, we have $\Pi\left(B_{1}, t\right)=C_{1} \in M, I\left(C_{1}\right)=B_{2} \in$ $N$, then $B_{2}$ is defined as the successor point of $B_{1}$, and $g\left(B_{1}\right)=y_{B_{2}}-y_{B_{1}}$ is defined as the successor function of point $B_{1}$. 


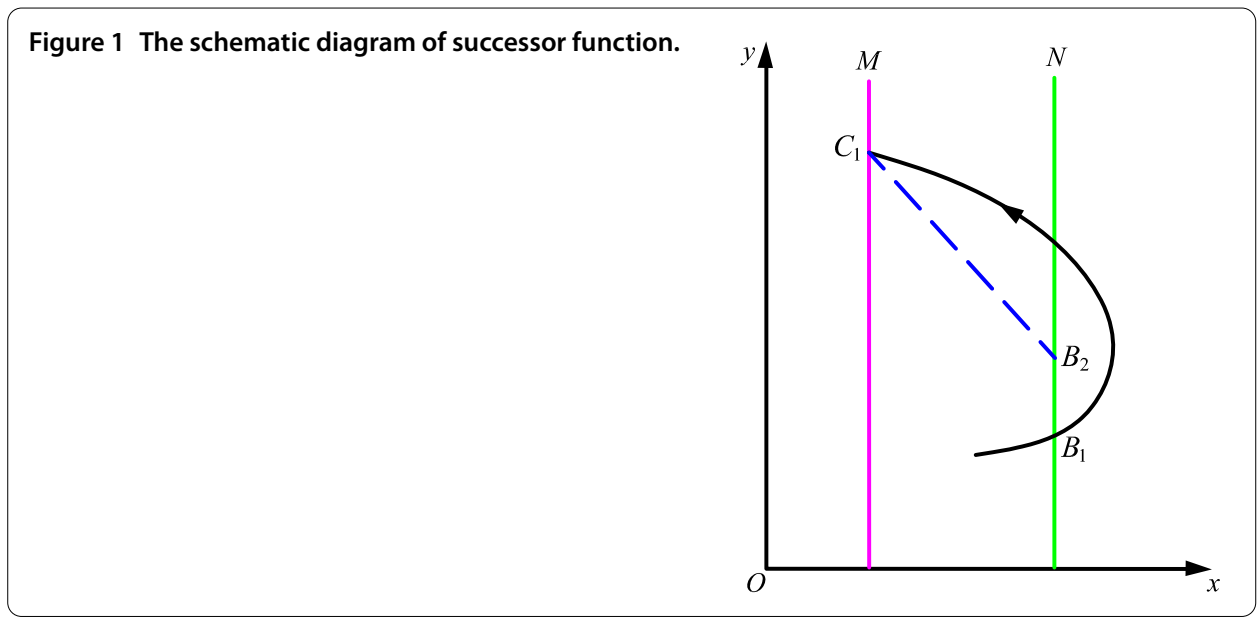

Lemma 2.1 ([47]) Successor function $g\left(B_{1}\right)$ is continuous.

Lemma 2.2 ([48]) In system (4), if there exist $A \in N, B \in N$ satisfying successor function $g(A) g(B)<0$, then there must exist a point $S(S \in N)$ satisfying $S$ between point $A$ and point $B$ such that $g(S)=0$, thus system (4) has an order-1 periodic solution.

\section{Dynamical analysis of system (2)}

The dynamical properties of the order-1 periodic solution of system (2) are mainly investigated in this section. Before these discussions, we firstly analyze the qualitative characteristics of system (2) without control, and the conditions that system (2) without control has no closed orbit are discussed.

\subsection{Qualitative analysis of system (2) without control}

Consider the continuous system of system (2) without control as follows:

$$
\left\{\begin{array}{l}
x^{\prime}(t)=a x(t)\left(1-\frac{x(t)}{K}\right)-b x(t) y(t)=P(x, y) \\
y^{\prime}(t)=y(t)(\lambda b x(t)-d)-H=Q(x, y) .
\end{array}\right.
$$

By setting

$$
\left\{\begin{array}{l}
a x(t)\left(1-\frac{x(t)}{K}\right)-b x(t) y(t)=0 \\
y(t)(\lambda b x(t)-d)-H=0
\end{array}\right.
$$

we have

$$
\frac{a \lambda}{K} x^{2}-\left(a \lambda+\frac{a d}{b K}\right) x+\frac{a d}{b}+H=0 .
$$

Let

$$
\Delta=\left(a \lambda+\frac{a d}{b K}\right)^{2}-4 \frac{a \lambda}{K}\left(\frac{a d}{b}+H\right),
$$


thus we find that if the condition

$$
\left(H_{1}\right):\left(\lambda+\frac{d}{b K}\right)^{2}>4 \frac{\lambda}{a K}\left(\frac{a d}{b}+H\right)
$$

holds, then the system (5) has two positive equilibria which are denoted as $E_{1}\left(x_{E_{1}}, y_{E_{1}}\right)$ and $E_{2}\left(x_{E_{2}}, y_{E_{2}}\right)$, where

$$
\begin{array}{ll}
x_{E_{1}}=K \frac{a\left(\lambda+\frac{d}{b K}\right)-\sqrt{\Delta}}{2 a \lambda}=\frac{K}{2}+\frac{d}{2 \lambda b}-\frac{K \sqrt{\Delta}}{2 a \lambda}, & y_{E_{1}}=\frac{a}{b}\left(1-\frac{x_{E_{1}}}{K}\right), \\
x_{E_{2}}=K \frac{a\left(\lambda+\frac{d}{b K}\right)+\sqrt{\Delta}}{2 a \lambda}=\frac{K}{2}+\frac{d}{2 \lambda b}+\frac{K \sqrt{\Delta}}{2 a \lambda}, & y_{E_{2}}=\frac{a}{b}\left(1-\frac{x_{E_{2}}}{K}\right) .
\end{array}
$$

Next, the stability of these two equilibria are discussed. The Jacobian matrix at equilibrium $E_{i}, i=1,2$, is

$$
J\left(E_{i}\right)=\left(\begin{array}{cc}
a-\frac{2 a}{K} x_{E_{i}}-b y_{E_{i}} & -b x_{E_{i}} \\
\lambda b y_{E_{i}} & \lambda b x_{E_{i}}-d
\end{array}\right) .
$$

By calculations, we have

$$
\begin{aligned}
\operatorname{Det}\left(J\left(E_{i}\right)\right) & =\left(a-\frac{2 a}{K} x_{E_{i}}-b y_{E_{i}}\right)\left(\lambda b x_{E_{i}}-d\right)+\lambda b^{2} x_{E_{i}} y_{E_{i}} \\
& =\frac{2 a b \lambda x_{E_{i}}}{K}\left(\frac{K}{2}+\frac{d}{2 \lambda b}-x_{E_{i}}\right) \\
\operatorname{Tr}\left(J\left(E_{i}\right)\right)= & a-\frac{2 a}{K} x_{E_{i}}-b y_{E_{i}}+\lambda b x_{E_{i}}-d \\
= & \left(\lambda b-\frac{a}{K}\right) x_{E_{i}}-d .
\end{aligned}
$$

It is easy to see that $\operatorname{Det}\left(J\left(E_{1}\right)\right)>0$ and $\operatorname{Det}\left(J\left(E_{2}\right)\right)<0$, thus $E_{1}\left(x_{E_{1}}, y_{E_{1}}\right)$ is an elementary but not saddle-type positive equilibrium, and $E_{2}\left(x_{E_{2}}, y_{E_{2}}\right)$ is a saddle.

On the other hand, if the condition

$$
\left(H_{2}\right): \quad \lambda b-\frac{a}{K}<0
$$

holds, then $\operatorname{Tr}\left(J\left(E_{1}\right)\right)<0$, which means $E_{1}\left(x_{E_{1}}, y_{E_{1}}\right)$ is a locally asymptotically stable focus or node.

In the following, let Dulac function $B=x^{-1}$, then we get

$$
\begin{aligned}
D & =\frac{\partial(B P)}{\partial x}+\frac{\partial(B Q)}{\partial y} \\
& =\lambda b-\frac{a}{K}-d x^{-1}<0 .
\end{aligned}
$$

According to the Bendixson-Dulac theorem, the closed orbit of system (5) does not exist in the plane $R_{+}^{2}$. In conclusion, the following theorem is obtained. 


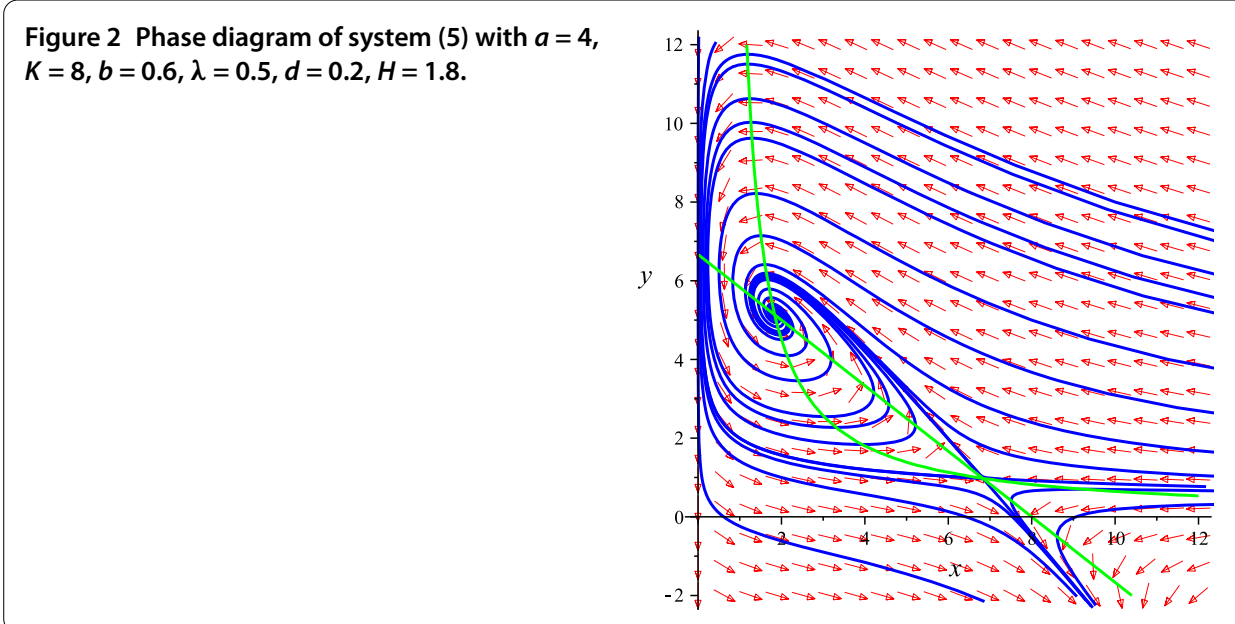

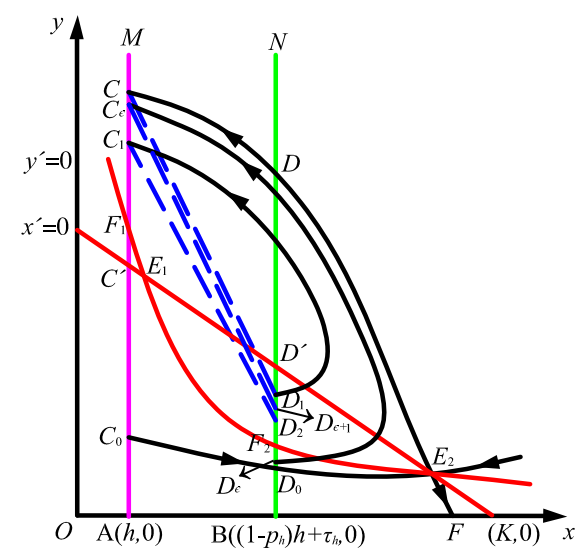

(a)

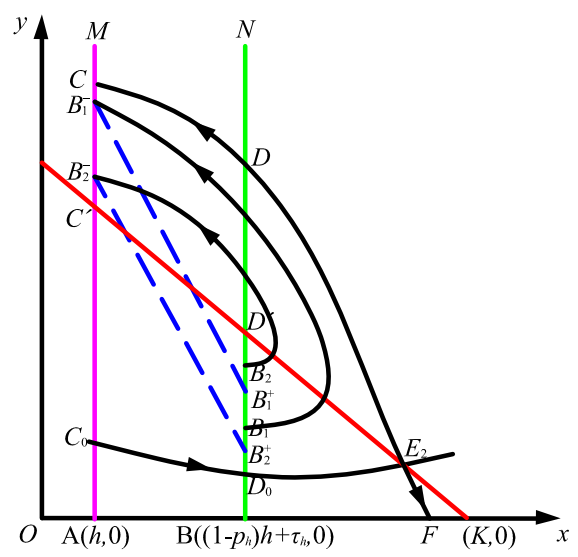

(b)

Figure 3 The existence and uniqueness of the order-1 periodic solution of system (2) in Case I. (a) The existence of the periodic solution. (b) The uniqueness of the periodic solution.

Theorem 3.1 System (5) has two positive equilibrium: a locally asymptotically stable focus or node $E_{1}\left(x_{E_{1}}, y_{E_{1}}\right)$ and a saddle $E_{2}\left(x_{E_{2}}, y_{E_{2}}\right)$, and there is no closed trajectory in the plane $R_{+}^{2}$ if the conditions $\left(H_{1}\right)$ and $\left(H_{2}\right)$ hold (see Figure 2 ).

\subsection{Existence, uniqueness and stability of order-1 periodic solution}

According to ecological significance, system (2) should satisfy $0<h<\left(1-p_{h}\right) h+\tau_{h}<K$. By the discussion in the previous subsection, we have the $x$-isoline $x^{\prime}=0$ intersects $y$-isoline $y^{\prime}=0$ at point $E_{1}\left(x_{E_{1}}, y_{E_{1}}\right)$ and point $E_{2}\left(x_{E_{2}}, y_{E_{2}}\right)$ (see Figure 3(a)). For notation simplicity, let the $x$-axis intersect the line $x=h$ (impulse set $M$ ) at point $A(h, 0)$ and intersect the line $x=\left(1-p_{h}\right) h+\tau_{h}$ (phase set $\left.N\right)$ at point $B\left(\left(1-p_{h}\right) h+\tau_{h}, 0\right)$, the $x$-isoline $x^{\prime}=0$ intersect the lines $x=h$ and $x=\left(1-p_{h}\right) h+\tau_{h}$ at points $C^{\prime}$ and $D^{\prime}$, respectively, the $y$-isoline $y^{\prime}=0$ intersect the lines $x=h$ and $x=\left(1-p_{h}\right) h+\tau_{h}$, respectively, at points $F_{1}$ and $F_{2} . C, D$ and $F$ are, respectively, the intersections between the stable flow of $E_{2}\left(x_{E_{2}}, y_{E_{2}}\right)$ and the line $x=h$, the line $x=\left(1-p_{h}\right) h+\tau_{h}, x$-axis. $C_{0}$ and $D_{0}$ are, respectively, the intersections between the unstable flow of $E_{2}\left(x_{E_{2}}, y_{E_{2}}\right)$ and the line $x=h$, the line $x=\left(1-p_{h}\right) h+\tau_{h}$. 
Theorem 3.2 System (2) exists a unique order-1 periodic solution if the conditions $\left(H_{1}\right)$, $\left(H_{2}\right)$ and $0<h<\left(1-p_{h}\right) h+\tau_{h}<K$ hold.

Proof For different threshold $h$, let us consider three cases as follows.

Case I. $0<h<x_{E_{1}}<\left(1-p_{h}\right) h+\tau_{h}<x_{E_{2}}$.

There exists a threshold $h \in\left(0, x_{E_{1}}\right)$ such that $q_{h} \in\left[q_{\min }, q_{\max }\right]$, due to impulsive effects, point $C$ jumps to a point $D_{1} \in \overline{D_{0} D^{\prime}} \subset N$, then $y_{D_{0}}<y_{D_{1}}=\left(1-q_{h}\right) y_{C}<y_{D^{\prime}}$. Besides, the orbit of system (2) starting from point $D_{1}$ must pass through a point $C_{1} \in M$, then jumps back to a point $D_{2} \in N$. Because distinct orbits are disjoint, then $y_{C^{\prime}}<y_{C_{1}}<y_{C}$ and $y_{D_{2}}=$ $\left(1-q_{h}\right) y_{C_{1}}<\left(1-q_{h}\right) y_{C}=y_{D_{1}}$, thus the successor function of point $D_{1}$ is $g\left(D_{1}\right)=y_{D_{2}}-$ $y_{D_{1}}<0$.

Moreover, another point $D_{\epsilon} \in \overline{D_{0} D^{\prime}}$ is selected and satisfies $y_{D_{\epsilon}}=y_{D_{0}}+\epsilon(\epsilon>0$ sufficiently small). There must be an orbit starting from point $D_{\epsilon}$ and passing through point $C_{\epsilon} \in M$, and point $C_{\epsilon}$ is next to point $C$, due to impulsive effects, point $C_{\epsilon}$ jumps to a point $D_{\epsilon+1} \in N$. Because distinct orbits are disjoint, we know $y_{C_{1}}<y_{C_{\epsilon}}<y_{C}$ and $y_{D_{\epsilon}}<y_{D_{2}}=\left(1-q_{h}\right) y_{C_{1}}<\left(1-q_{h}\right) y_{C_{\epsilon}}=y_{D_{\epsilon+1}}$. Then we have $g\left(D_{\epsilon}\right)=y_{D_{\epsilon+1}}-y_{D_{\epsilon}}>0$.

We can easily get $g\left(D_{1}\right) g\left(D_{\epsilon}\right)<0$, there is a point $S \in \overline{D_{\epsilon} D_{1}}$ such that $f(S)=0$ by Lemma 2.2, i.e. the order-1 periodic solution is existent.

In the following, the uniqueness of the order-1 periodic solution is proved. Arbitrarily select two points $B_{1}$ and $B_{2}$ in the line $x=(1-p) h_{h}+\tau_{h}$ which meet $y_{D_{0}}<y_{B_{1}}<y_{B_{2}}<y_{D^{\prime}}$ (see Figure 3(b)). The orbits of system (2) starting from points $B_{1}$ and $B_{2}$, respectively, reach points $B_{1}^{-} \in M$ and $B_{2}^{-} \in M$, and satisfy $y_{C^{\prime}}<y_{B_{2}^{-}}<y_{B_{1}^{-}}<y_{C}$, then jump back to the line $x=\left(1-p_{h}\right) h+\tau_{h}$ at $B_{1}^{+}$and $B_{2}^{+}$by impulsive effects, respectively. Then the successor functions of points $B_{1}$ and $B_{2}$ must satisfy

$$
\begin{aligned}
g\left(B_{2}\right)-g\left(B_{1}\right) & =\left(y_{B_{2}^{+}}-y_{B_{2}}\right)-\left(y_{B_{1}^{+}}-y_{B_{1}}\right) \\
& =\left(y_{B_{2}^{+}}-y_{B_{1}^{+}}\right)+\left(y_{B_{1}}-y_{B_{2}}\right)<0,
\end{aligned}
$$

which illustrates the successor function $g$ in the segment $\overline{D_{0} D^{\prime}}$ is monotonically decreasing, thus there is only one point $S \in \overline{D_{0} D^{\prime}}$ that makes $g(S)=0$.

For any point $S_{1} \in \overline{D D^{\prime}}$, the orbit of system (2) starting from point $S_{1}$ intersects a point in the line $x=h$ which is denoted as $S_{1}^{-}$, then jumps to a point $S_{1}^{+} \in N$ after impulsive effects. Because distinct orbits are disjoint, then $y_{C^{\prime}}<y_{S_{1}^{-}}<y_{C}$ and $y_{S_{1}^{+}}=\left(1-q_{h}\right) y_{S_{1}^{-}}<\left(1-q_{h}\right) y_{C}=$ $y_{D_{1}}<y_{S_{1}}$, thus we get $g\left(S_{1}\right)=y_{S_{1}^{+}}-y_{S_{1}}<0$, which says there is no order-1 periodic orbit passing through point $S_{1} \in \overline{D D^{\prime}}$. In addition, for any point $S_{2} \in \overline{B D_{0}}$, the orbit starting from point $S_{2}$ eventually passes through the line $y=0$ and unaffected by any impulse, namely, there is no order-1 periodic orbit passing through point $S_{2}$.

Case II. $x_{E_{1}} \leq h<\left(1-p_{h}\right) h+\tau_{h} \leq x_{E_{2}}$.

The following steps are similar to the Case I and omitted thereby (see Figure 4).

Case III. $x_{E_{1}}<h<x_{E_{2}}<\left(1-p_{h}\right) h+\tau_{h}<K$.

For this subcase, the stable flow of $E_{2}$ intersects the line $x=(1-p) h_{h}+\tau_{h}$ at point $G_{1}$, and the unstable flow of $E_{2}$ intersects the line $x=(1-p) h_{h}$ at point $H_{1}$. We can select a point $G_{\epsilon}$ satisfying $y_{G_{\epsilon}}=y_{G_{1}}+\epsilon$, there must exist an orbit starting from point $G_{\epsilon}$ and passing through point $H_{\epsilon} \in M$, and point $H_{\epsilon}$ is next to point $H_{1}$. By impulsive effects, point $H_{\epsilon}$ jumps to a point $G_{\epsilon+1} \in N$ which is above $G_{\epsilon}$. Then $g\left(G_{\epsilon}\right)=y_{G_{\epsilon+1}}-y_{G_{\epsilon}}>0$. 


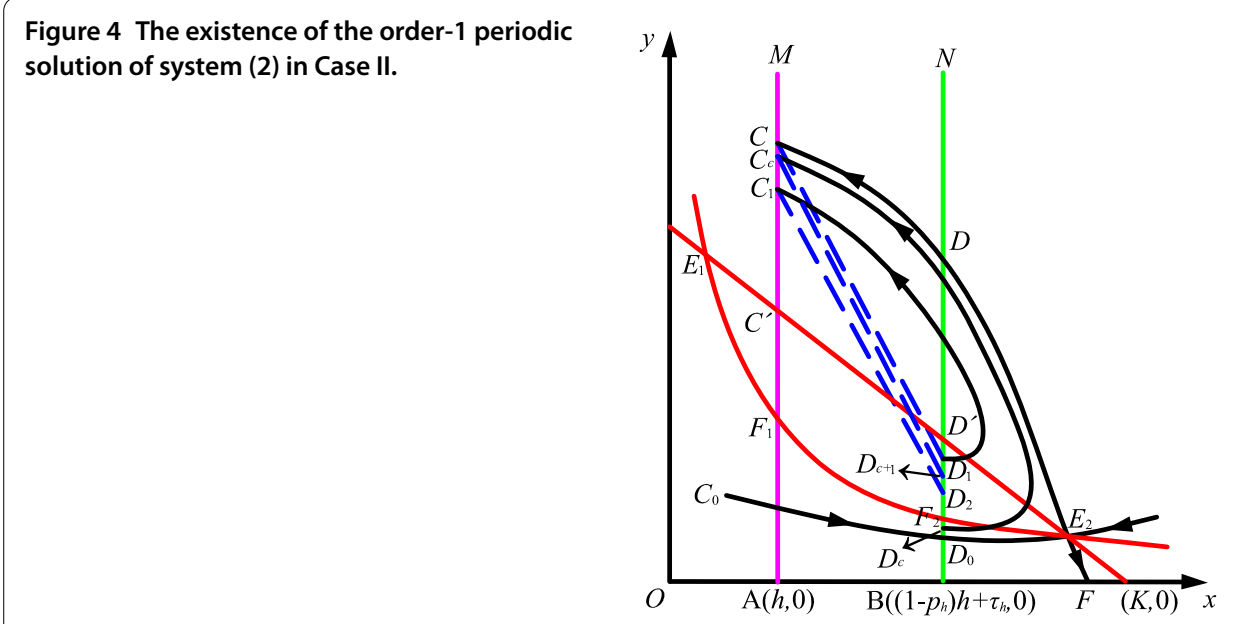

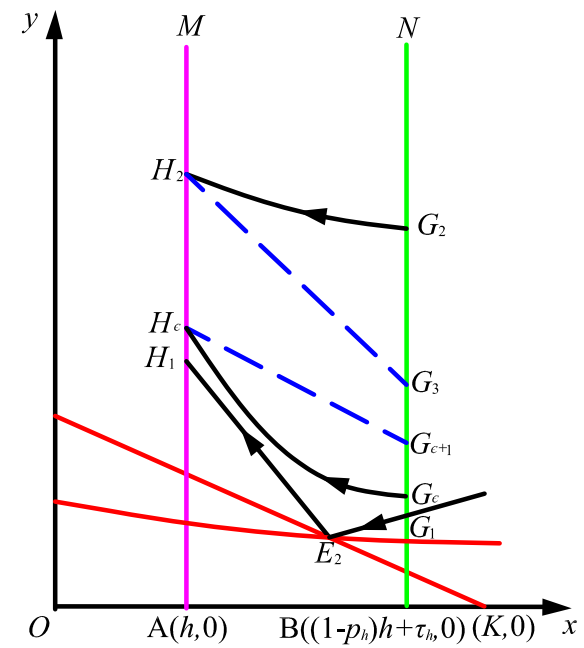

(a)

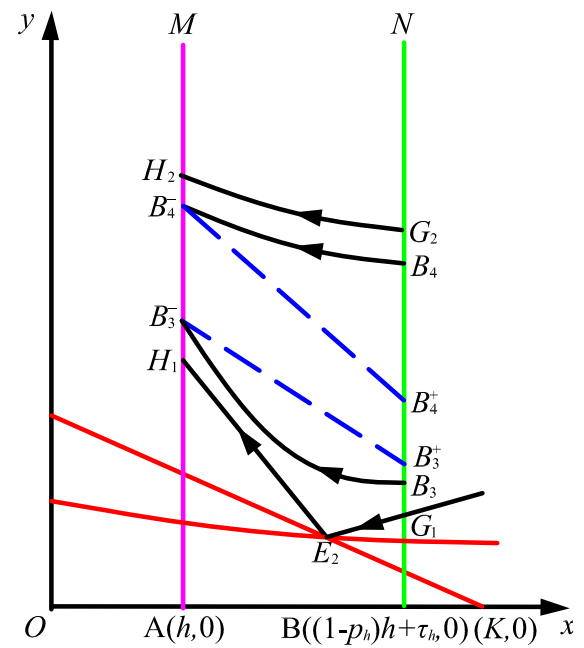

(b)

Figure 5 The existence and uniqueness of the order-1 periodic solution of system (2) in Case III. (a) The existence of the periodic solution. (b) The uniqueness of the periodic solution.

Furthermore, we can select another orbit that is far from the stable flow and unstable flow of $E_{2}$ which passes through point $G_{2} \in N$, and reaches point $H_{2} \in M$, then jumps back to the line $x=\left(1-p_{h}\right) h+\tau_{h}$ at point $G_{3}$, and point $G_{3}$ is below point $G_{2}$, then $g\left(G_{2}\right)=$ $y_{G_{3}}-y_{G_{2}}<0$.

We can easily get $g\left(G_{\epsilon}\right) g\left(G_{2}\right)<0$. Then there is a point $S \in \overline{G_{\epsilon} G_{2}}$ such that $g(S)=0$, namely, the order-1 periodic solution is existent (see Figure 5(a)).

Next, we prove the uniqueness of the periodic solution. From Figure 5(b), arbitrarily select two points $B_{3} \in N$ and $B_{4} \in N$ which meet $y_{G_{1}}<y_{B_{3}}<y_{B_{4}}<y_{G_{2}}$. The orbits of system (2) starting from points $B_{3}$ and $B_{4}$ respectively reach at points $B_{3}^{-} \in M$ and $B_{4}^{-} \in M$, and satisfy $y_{H_{1}}<y_{B_{3}^{-}}<y_{B_{4}^{-}}<y_{H_{2}}$, then jump back to the line $x=\left(1-p_{h}\right) h+\tau_{h}$ at $B_{3}^{+}$and $B_{4}^{+}$due to impulsive effects, respectively. Point $B_{3}^{+}$is above $B_{3}$ and point $B_{4}^{+}$is blow $B_{4}$. Then the 
successor functions of points $B_{3}$ and $B_{4}$ must satisfy

$$
g\left(B_{4}\right)-g\left(B_{3}\right)=\left(y_{B_{4}^{+}}-y_{B_{4}}\right)-\left(y_{B_{3}^{+}}-y_{B_{3}}\right)<0,
$$

which illustrates, in the segment $\overline{G_{1} G_{2}}$, the successor function $f$ is monotonically decreasing, thus there is only one point $S \in \overline{G_{1} G_{2}}$ that makes $g(S)=0$.

For any point $S_{3} \in \overline{G_{1} B}$, the orbit starting from point $S_{3}$ eventually passes through the line $y=0$ and unaffected by any impulse, namely, there is no order-1 periodic orbit passing through point $S_{3} \in \overline{G_{1} B}$.

In this paper, we assume that the order-1 periodic solution of system (2) is $\widehat{S S^{-} S}$, where $S \in N$ and $S^{-} \in M$. Next we prove the stability of the periodic solution $\widehat{S S^{-} S}$. Since the methods used in the above three cases are similar, we only prove Case II.

Theorem 3.3 The periodic solution $\widehat{S S^{-S}}$ is orbitally asymptotically stable if $x_{E_{1}}<h<(1-$ $\left.p_{h}\right) h+\tau_{h}<x_{E_{2}}$ and $\frac{a}{b}\left(1-q_{h}\right)\left(1-\frac{h}{K}\right) \geq \frac{H}{\lambda b\left[\left(1-p_{h}\right) h+\tau_{h}\right]-d}$ hold under Theorem 3.2.

Proof From Figure 4 we can see $y_{F_{2}}=\frac{H}{\lambda b\left[\left(1-p_{h}\right) h+\tau_{h}\right]-d}>y_{D_{0}}$. Besides, by $y_{C^{\prime}}=\frac{a}{b}\left(1-\frac{h}{K}\right)$ and $\frac{a}{b}\left(1-q_{h}\right)\left(1-\frac{h}{K}\right) \geq \frac{H}{\lambda b\left[\left(1-p_{h}\right) h+\tau_{h}\right]-d}$, it is easy to know $\left(1-q_{h}\right) y_{C^{\prime}} \geq y_{F_{2}}>y_{D_{0}}$, then, for any point $S_{4} \in \overline{C C^{\prime}}$, we get $\left(1-q_{h}\right) y_{S_{4}} \geq\left(1-q_{h}\right) y_{C^{\prime}} \geq y_{F_{2}}>y_{D_{0}}$. We know the periodic solution $\widehat{S S^{-S}}$ is unique, where $S \in \overline{D_{0} D_{1}}$. From Figure 6 we can see the orbit of system (2) starting from $D_{1}$ intersects the line $x=h$ at point $C_{1}$, then jumps bake to the line $x=\left(1-p_{h}\right) h+\tau_{h}$ at point $D_{2}$ due to impulsive effects. Because distinct orbits are disjoint, we have $y_{C^{\prime}}<$ $y_{C_{1}}<y_{S^{-}}$and $y_{D_{0}}<y_{D_{2}}<y_{S}$. The orbit starting from $D_{2}$ intersects the line $x=h$ at point $C_{2}$, then jumps bake to the line $x=\left(1-p_{h}\right) h+\tau_{h}$ at point $D_{3}$ after impulsive effects, where $y_{S^{-}}<y_{C_{2}}<y_{C}$ and $y_{S}<y_{D_{3}}<y_{D_{1}}$.

Repeat the above process, the orbit starting from point $D_{0}$ will be subjected to impulsive effects infinitely times. Denote the successor point of point $D_{i}$ as $D_{i+1}, i=0,1,2, \ldots$, then we get

$$
y_{D_{0}}<y_{D_{2}}<y_{D_{4}}<\cdots<y_{D_{2 i}}<y_{D_{2(i+1)}}<\cdots<y_{S}
$$

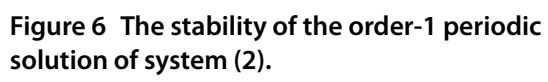


and

$$
y_{D_{1}}>y_{D_{3}}>y_{D_{5}}>\cdots>y_{D_{2 i+1}}>y_{D_{2(i+1)+1}}>\cdots>y_{S} .
$$

Therefore, the sequence $\left\{D_{2 i}\right\}$ is monotonically increasing and the sequence $\left\{D_{2 i+1}\right\}$ is monotonically decreasing. Besides,

$$
y_{D_{2 i}} \rightarrow y_{S}, \quad \text { as } i \rightarrow \infty,
$$

and

$$
y_{D_{2 i+1}} \rightarrow y_{S}, \quad \text { as } i \rightarrow \infty .
$$

We select any point $P_{0} \in \overline{D_{0} D_{1}}$ and let $y_{D_{0}}<y_{P_{0}}<y_{S}$ (otherwise, $y_{D_{1}}>y_{P_{0}}>y_{S}$, the proofs are similar), then there must be a positive integer $k_{0}$ which satisfy $y_{D_{2 k_{0}}}<y_{P_{0}}<y_{D_{2\left(k_{0}+1\right)}}$. The orbit starting from point $P_{0}$ will be affected by impulse infinitely times. Affected by the $j$ th impulse, the corresponding phase point is denoted as $P_{j}, j=1,2, \ldots$, then, for any $n$, we get $y_{D_{2\left(k_{0}+n\right)}}<y_{P_{2 n}}<y_{D_{2\left(k_{0}+n+1\right)}}$ and $y_{D_{2\left(k_{0}+n+1\right)}}<y_{P_{2 n+1}}<y_{D_{2\left(k_{0}+n\right)+1}}, n=0,1,2, \ldots$, thus $\left\{y_{P_{2 n}}\right\}$ is monotonically increasing, and $\left\{y_{P_{2 n+1}}\right\}$ is monotonically decreasing, and

$$
y_{D_{2 n}} \rightarrow y_{S}, \quad \text { as } n \rightarrow \infty,
$$

and

$$
y_{D_{2 n+1}} \rightarrow y_{S}, \quad \text { as } n \rightarrow \infty .
$$

Therefore, all the successor points in the segment $\overline{D^{\prime} D_{0}}$ are attracted to point $S$ after the corresponding impulsive effect, then the periodic solution $\widehat{S S^{-} S}$ is orbitally asymptotically stable. That completes the proof.

\section{Simulations and optimization}

\subsection{Numerical simulations}

A specific model is given in this subsection to verify the effectiveness of our conclusions. Let $a=4, K=8, b=0.6, \lambda=0.5, d=0.2, H=1.8, h_{\max }=6, h_{\min }=1.3, p_{\max }=0.01, p_{\min }=$ $0.001, \tau_{\max }=1.4, \tau_{\min }=1.27, q_{\max }=0.7, q_{\min }=0.52$. By calculation, the equilibrium points of system (5) are $E_{1}(1.8344,5.1380)$ and $E_{2}(6.8322,0.9731)$. These parameter values are substituted into system (2), then we find

$$
\left\{\begin{array}{l}
x^{\prime}(t)=4 x(t)\left(1-\frac{x(t)}{8}\right)-0.6 x(t) y(t), \\
y^{\prime}(t)=y(t)(0.3 x(t)-0.2)-1.8, \\
\Delta x(t)=-p(x) x(t)+\tau(x), \\
\Delta y(t)=-q(x) y(t),
\end{array}\right\} \quad x>h,
$$

Let $h=1.5$ satisfy the condition $0<h<x_{E_{1}}$, we select the orbit starting from $(4,4)$. A directed calculation yields $p_{1.5}=0.0096, \tau_{1.5}=1.3945$ and $q_{1.5}=0.6923$ which satisfy the condition $h<x_{E_{1}}<\left(1-p_{h}\right) h+\tau_{h}<x_{E_{2}}$. Then there exists an order-1 periodic solution in system (7) which is unique and asymptotically stable; see Figures 7(a), 7(b) and 7(c). 

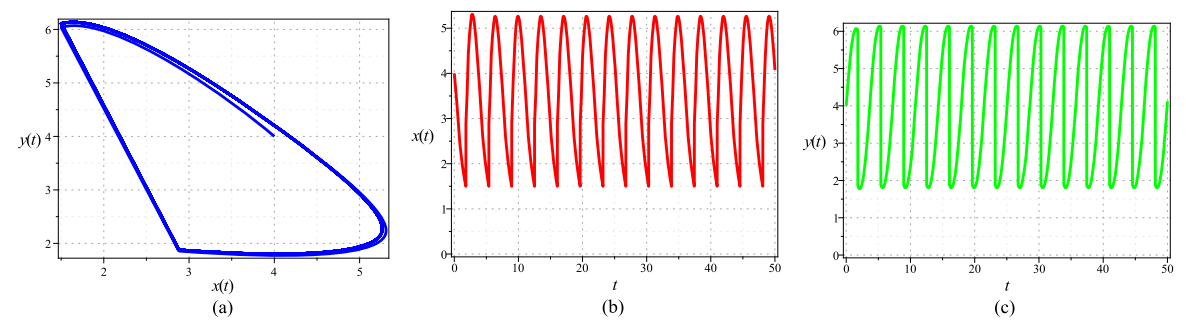

Figure 7 Numerical simulations in the case $0<\boldsymbol{h}<\boldsymbol{x}_{E_{1}}<\left(\mathbf{1}-\boldsymbol{p}_{\boldsymbol{h}}\right) \boldsymbol{h}+\boldsymbol{\tau}_{\boldsymbol{h}}<\boldsymbol{x}_{E_{2}}$. (a) Phase portrait of prey fish density and predator fish density on $h=1.5$. (b) Time series of prey fish density. (c) Time series of predator fish density.

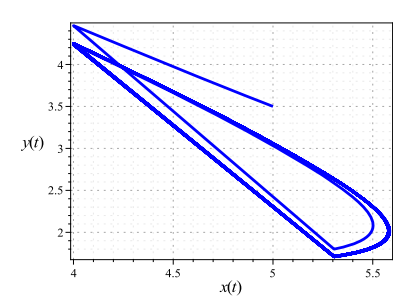

(a)

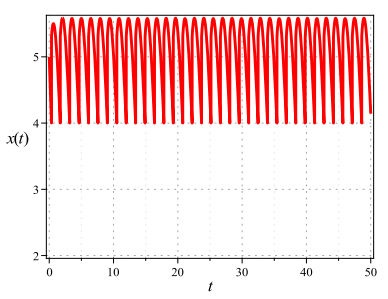

(b)

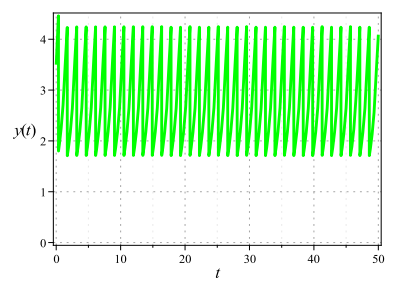

(c)

Figure 8 Numerical simulations in the case $x_{E_{1}}<\boldsymbol{h}<\left(1-p_{h}\right) \boldsymbol{h}+\boldsymbol{\tau}_{\boldsymbol{h}}<\boldsymbol{x}_{E_{2}}$. (a) Phase portrait of prey fish density and predator fish density on $h=4$. (b) Time series of prey fish density. (c) Time series of predator fish density.
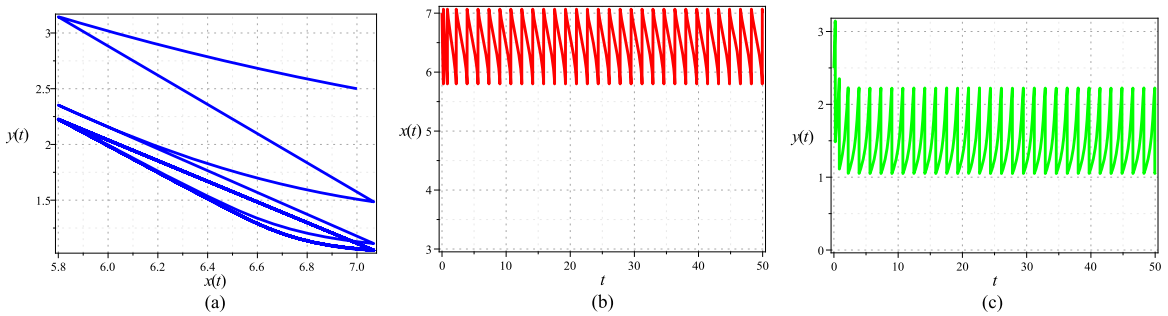

Figure 9 Numerical simulations in case $x_{E_{1}}<\boldsymbol{h}<\boldsymbol{x}_{E_{2}}<\left(1-p_{h}\right) \boldsymbol{h}+\boldsymbol{\tau}_{\boldsymbol{h}}<\boldsymbol{K}$. (a) Phase portrait of prey fish density and predator fish density on $h=5.8$. (b) Time series of prey fish density. (c) Time series of predator fish density.

Furthermore, we obtain the period of order- 1 periodic solution is $T=3.5667$ by observing from Figure $7(\mathrm{~b})$.

The phase portrait and time series of prey fish density and predator fish density are shown in Figure 8 for $h=4$ with the initial value $(5,3.5)$, by calculation we obtain $p_{4}=$ $0.0048, \tau_{4}=1.3253$ and $q_{4}=0.5966$ which satisfy the condition $x_{E_{1}}<h<\left(1-p_{h}\right) h+\tau_{h}<$ $x_{E_{2}}$. Then system (7) exists a unique and orbitally asymptotically stable order-1 periodic solution, and the period is $T=1.4625$; see Figures $8(\mathrm{a}), 8(\mathrm{~b})$ and $8(\mathrm{c})$.

For the case of $x_{E_{1}}<h<x_{E_{2}}<\left(1-p_{h}\right) h+\tau_{h}<K$, for example $h=5.8$ and the orbit of system $(7)$ starting from $(7,2.5)$, by calculation, we get $p_{5.8}=0.0014, \tau_{5.8}=1.2755$ and $q_{5.8}=$ 0.5277 . Then system (7) exists an order-1 periodic solution which is unique and orbitally asymptotically stable and its period is $T=1.7083$; see Figures 9(a), 9(b) and 9(c). 


\subsection{Determination of optimal threshold $h$}

The practical significance of studying the order-1 periodic solution is that it provides the possibility to determine the replenishment rate of prey fish and the harvesting rate of predator fish, which makes the impulsive control to be not a real-time monitoring of fisheries, but rather a periodic one. In order to maintain the ecological balance of fisheries, further determine the optimal replenishment rate of prey fish and the optimal harvesting rate of predator fish, and make sure the harvest period is shortest and the profit is highest, we consider the following optimization problem to find the optimal threshold.

Let $l_{1}$ denote the unit cost of prey fish replenished including the cost of dealing with fisheries environment, $l_{2}$ be the unit income of predator fish. Our objective is to minimize costs and maximize profits in this process. Denote $F$ as the total profit in one period of system (7), which is a function of replenishment rate of prey fish $\tau_{h}$ and the harvesting rate of predator fish $q_{h}$. Since $H$ is constant and has no effect on the change in profits, then we no longer consider it and have $F(h)=l_{2} q_{h}-l_{1} \tau_{h}$. Thus, the optimization model is formulated as

$$
\begin{aligned}
& \max \frac{F(h)}{T(h)} \\
& \text { s.t. } h_{\min } \leq h \leq h_{\max }
\end{aligned}
$$

The optimization problem is solved to yield the optimal threshold $h^{*}$, which results in the optimal replenishment rate of prey fish $\tau^{*}=p_{h^{*}}$, the optimal harvesting rate of predator fish $q^{*}=q_{h^{*}}$, and the optimal impulse period $T^{*}=T\left(\tau^{*}, q^{*}\right)$. The impulse period $T$ varies with the threshold $h$, as shown in Figure 10(a), and the relationship between the profit per unit time $F / T$ and the threshold $h$ is presented in Figure 10(b), where $l_{1}=200$, $l_{2}=5000$, i.e., $l_{2} / l_{1}=25$. From Figure 10, the optimal threshold is $h^{*}=5$, then the optimal replenishment rate of prey fish $\tau^{*}=1.2977$, the optimal harvesting rate of predator fish $q^{*}=0.5583$, and the optimal impulse period is $T^{*}=1.2767$.

\section{Conclusion}

This work presents a prey-predator system with both state-dependent impulsive harvesting and constant rate harvesting, where the harvesting frequency of constant harvesting

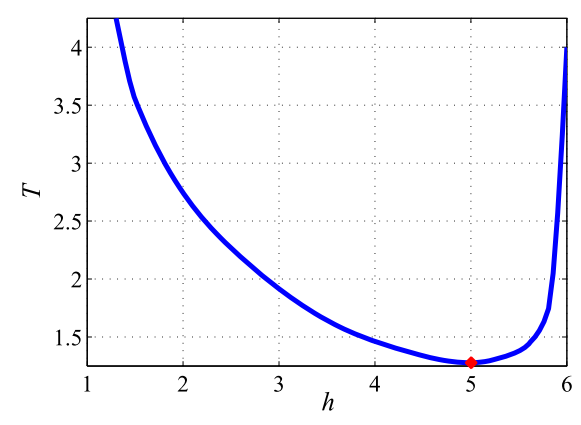

(a)

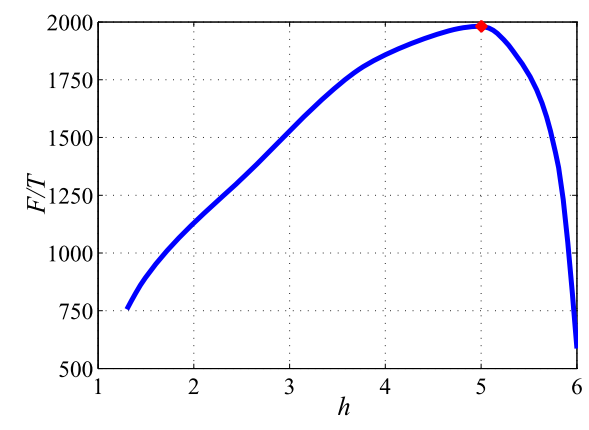

(b)

Figure 10 The variety in the period $T$ and the profit per unit time $F / T$ on the threshold $h$. (a) The variety in the period $T$ on the threshold $h$. (b) The profit per unit time $F / T$ on the threshold $h$. 
is more frequent than that of impulse harvesting. Moreover, the combination of these two harvesting methods is more practical which provides higher commercial value and avoids the exhaustion of resources. Meanwhile, the existence, uniqueness and stability of the order-1 periodic solution are proved by using the method of successor functions and differential equation geometry theory. Numerical simulations with a specific example are given to verify feasibility of the impulsive strategy. Furthermore, to maximize economic benefit, we provide an optimization strategy for the pisciculture and obtain the optimal threshold. However, the optimization results have some deviations which need to be further improved.

\section{Acknowledgements}

The paper is supported in part by the National Natural Science Foundation of China (No. 11371230, 11501331), in part by Shandong Provincial Natural Science Foundation, China (No. S2015SF002), in part by SDUST Research Fund (2014TDJH102), and in part by Joint Innovative Center for Safe and Effective Mining Technology and Equipment of Coal Resources, Shandong Province of China.

\section{Competing interests}

The authors claim that they have no competing interests.

\section{Authors' contributions}

All authors read and approved the final manuscript.

\section{Author details}

${ }^{1}$ College of Mathematics and Systems Science, Shandong University of Science and Technology, Qingdao, China. ${ }^{2}$ State Key Laboratory of Mining Disaster Prevention and Control Co-founded by Shandong Province and the Ministry of Science and Technology, Shandong University of Science and Technology, Qingdao, China.

\section{Publisher's Note}

Springer Nature remains neutral with regard to jurisdictional claims in published maps and institutional affiliations.

Received: 27 October 2017 Accepted: 18 January 2018 Published online: 25 January 2018

\section{References}

1. Pal, D, Mahapatra, GS: A bioeconomic modeling of two-prey and one-predator fishery model with optimal harvesting policy through hybridization approach. Appl. Math. Comput. 242, 748-763 (2014)

2. Liu, G, Wang, X, Meng, X, Gao, S: Extinction and persistence in mean of a novel delay impulsive stochastic infected predator-prey system with jumps. Complexity 2017(3), Article ID 1950970 (2017)

3. Liu, L, Meng, X: Optimal harvesting control and dynamics of two-species stochastic model with delays. Adv. Differ. Equ. 2017(1), 18 (2017)

4. Bian, F, Zhao, W, Song, Y, Yue, R: Dynamical analysis of a class of prey-predator model with Beddington-Deangelis functional response, stochastic perturbation, and impulsive toxicant input. Complexity 2017, Article ID 3742197 (2017)

5. Brauer, F, Soudack, AC: Stability regions in predator-prey systems with constant-rate prey harvesting. J. Math. Biol. 8(1), 55-71 (1979)

6. Martin, A, Ruan, S: Predator-prey models with delay and prey harvesting. J. Math. Biol. 43(3), 247-267 (2001)

7. Xiao, D, Li, W, Han, M: Dynamics in a ratio-dependent predator-prey model with predator harvesting. J. Math. Anal. Appl. 324(1), 14-29 (2006)

8. Chen, L, Chen, F: Global analysis of a harvested predator-prey model incorporating a constant prey refuge. Int. J. Biomath. 3(02), 205-223 (2010)

9. Zhang, T, Ma, W, Meng, X: Global dynamics of a delayed chemostat model with harvest by impulsive flocculant input. Adv. Differ. Equ. 2017(1), 115 (2017)

10. Wang, Y, Jiang, W, Wang, H: Stability and global Hopf bifurcation in toxic phytoplankton-zooplankton model with delay and selective harvesting. Nonlinear Dyn. 73(1), 881-896 (2013)

11. Meng, X, Wang, L, Zhang, T: Global dynamics analysis of a nonlinear impulsive stochastic chemostat system in a polluted environment. J. Appl. Anal. Comput. 6(3), 865-875 (2016)

12. Zhang, $T$, Meng, $X$, Song, Y: The dynamics of a high-dimensional delayed pest management model with impulsive pesticide input and harvesting prey at different fixed moments. Nonlinear Dyn. 64(1), 1-12 (2011)

13. Jiang, Z, Wang, L: Global Hopf bifurcation for a predator-prey system with three delays. Int. J. Bifurc. Chaos 27(07), 1750108 (2017)

14. Zhang, S, Meng, X, Feng, T, Zhang, T: Dynamics analysis and numerical simulations of a stochastic non-autonomous predator-prey system with impulsive effects. Nonlinear Anal. Hybrid Syst. 26, 19-37 (2017)

15. Meng, X, Zhang, L: Evolutionary dynamics in a Lotka-Volterra competition model with impulsive periodic disturbance. Math. Methods Appl. Sci. 39(2), 177-188 (2016)

16. Zhang, T, Ma, W, Meng, X: Impulsive control of a continuous-culture and flocculation harvest chemostat model. Int. J. Syst. Sci. 48(16), 3459-3469 (2017) 
17. Pei, Y, Li, C, Chen, L: Continuous and impulsive harvesting strategies in a stage-structured predator-prey model with time delay. Math. Comput. Simul. 79(10), 2994-3008 (2009)

18. Jiao, J, Cai, S, Li, L: Dynamics of a periodic switched predator-prey system with impulsive harvesting and hibernation of prey population. J. Franklin Inst. 353(15), 3818-3834 (2016)

19. Huang, C, Meng, Y, Cao, J, Alsaedi, A, Alsaadi, FE: New bifurcation results for fractional BAM neural network with leakage delay. Chaos Solitons Fractals 100, 31-44 (2017)

20. Huang, C, Cao, J, Xiao, M, Alsaedi, A, Hayat, T: Effects of time delays on stability and Hopf bifurcation in a fractional ring-structured network with arbitrary neurons. Commun. Nonlinear Sci. Numer. Simul. 57, 1-13 (2018)

21. Huang, C, Cao, J, Xiao, M, Alsaedi, A, Hayat, T: Bifurcations in a delayed fractional complex-valued neural network. Appl. Math. Comput. 292, 210-227 (2017)

22. Huang, C, Cao, J, Xiao, M, Alsaedi, A, Alsaadi, FE: Controlling bifurcation in a delayed fractional predator-prey system with incommensurate orders. Appl. Math. Comput. 293, 293-310 (2017)

23. Zhang, T, Ma, W, Meng, X, Zhang, T: Periodic solution of a prey-predator model with nonlinear state feedback control. Appl. Math. Comput. 266, 95-107 (2015)

24. Jiao, J, Chen, L, Long, W: Pulse fishing policy for a stage-structured model with state-dependent harvesting. J. Biol. Syst. 15(03), 409-416 (2008)

25. Nie, L, Teng, Z, Hu, L, Peng, J: The dynamics of a Lotka-Volterra predator-prey model with state dependent impulsive harvest for predator. Biosystems 98(2), 67-72 (2009)

26. Huang, $M$, Song, $X$ : Periodic solutions and homoclinic bifurcations of two predator-prey systems with nonmonotonic functional response and impulsive harvesting. J. Appl. Math. 2014, Article ID 803764 (2014)

27. Wei, C, Chen, L: Periodic solution and heteroclinic bifurcation in a predator-prey system with Allee effect and impulsive harvesting. Nonlinear Dyn. 76(2), 1109-1117 (2014)

28. Yang, J, Tang, S: Holling type II predator-prey model with nonlinear pulse as state-dependent feedback control. J. Comput. Appl. Math. 291, 225-241 (2016)

29. Cheng, H, Zhang, T, Wang, F: Existence and attractiveness of order one periodic solution of a Holling I predator-prey model. Abstr. Appl. Anal. 2012, Article ID 126018 (2012)

30. Zhang, H, Georgescu, P, Zhang, L: Periodic patterns and Pareto efficiency of state dependent impulsive controls regulating interactions between wild and transgenic mosquito populations. Commun. Nonlinear Sci. Numer. Simul. 31(1), 83-107 (2016)

31. Cheng, H, Wang, F, Zhang, T: Multi-state dependent impulsive control for pest management. J. Appl. Math. 2012, Article ID 381503 (2012)

32. Tian, Y, Zhang, T, Sun, K: Dynamics analysis of a pest management prey-predator model by means of interval state monitoring and control. Nonlinear Anal. Hybrid Syst. 23, 122-141 (2017)

33. Zhang, T, Meng, X, Liu, R, Zhang, T: Periodic solution of a pest management Gompertz model with impulsive state feedback control. Nonlinear Dyn. 78(2), 921-938 (2014)

34. Miao, A, Zhang, J, Zhang, T, Pradeep, BGSA: Threshold dynamics of a stochastic SIR model with vertical transmission and vaccination. Comput. Math. Methods Med. 2017, 4820183 (2017)

35. Miao, A, Wang, X, Zhang, T, Wang, W, Pradeep, BGSA: Dynamical analysis of a stochastic SIS epidemic model with nonlinear incidence rate and double epidemic hypothesis. Adv. Differ. Equ. 2017, 226 (2017)

36. Zhao, W, Li, J, Meng, X: Dynamical analysis of SIR epidemic model with nonlinear pulse vaccination and lifelong immunity. Discrete Dyn. Nat. Soc. 2015, Article ID 848623 (2015)

37. Leng, X, Feng, T, Meng, X: Stochastic inequalities and applications to dynamics analysis of a novel SIVS epidemic model with jumps. J. Inequal. Appl. 2017, 138 (2017)

38. Lv, W, Wang, F: Adaptive tracking control for a class of uncertain nonlinear systems with infinite number of actuator failures using neural networks. Adv. Differ. Equ. 2017(1), 374 (2017)

39. LV, X, Wang, L, Meng, X: Global analysis of a new nonlinear stochastic differential competition system with impulsive effect. Adv. Differ. Equ. 2017(1), 296 (2017)

40. Huang, M, Liu, S, Song, X, Chen, L: Periodic solutions and homoclinic bifurcation of a predator-prey system with two types of harvesting. Nonlinear Dyn. 73, 815-826 (2013)

41. Xiao, Q, Dai, B, Xu, B, Bao, L: Homoclinic bifurcation for a general state-dependent Kolmogorov type predator-prey model with harvesting. Nonlinear Anal., Real World Appl. 26, 263-273 (2015)

42. Sun, K, Zhang, T, Tian, Y: Theoretical study and control optimization of an integrated pest management predator-prey model with power growth rate. Math. Biosci. 279, 13-26 (2016)

43. Chen, L: Pest control and geometric theory of semi-continuous dynamical system. J. Beihua Univ. Nat. Sci. 12(1), 1-9 (2011)

44. Liu, B, Tian, Y, Kang, B: Dynamics on a Holling II predator-prey model with state-dependent impulsive control. Int. J. Biomath. 5(03), 675 (2012)

45. Wang, J, Cheng, H, Meng, X, Pradeep, BSA: Geometrical analysis and control optimization of a predator-prey model with multi state-dependent impulse. Adv. Differ. Equ. 2017(1), 252 (2017)

46. Zhao, W, Liu, Y, Zhang, T, Meng, X: Geometric analysis of an integrated pest management model including two state impulses. Abstr. Appl. Anal. 2014(1), 91506 (2014)

47. Cheng, H, Wang, F, Zhang, T: Multi-state dependent impulsive control for Holling I predator-prey model. Discrete Dyn. Nat. Soc. 2012(12), 30-44 (2012)

48. Zhang, T, Zhang, J, Meng, X, Zhang, T: Geometric analysis of a pest management model with Holling's type III functional response and nonlinear state feedback control. Nonlinear Dyn. 84(3), 1529-1539 (2016) 